\title{
HEALTHY FUTURES Atlas: An Open-source WebGIS to Support Infectious Disease Intervention Planning in Eastern Africa
}

\author{
Lucia Morper-Busch $^{1}$, Stefan Kienberger ${ }^{2}$ and Michael Hagenlocher ${ }^{2}$ \\ ${ }^{1}$ University of Wuerzburg, Germany·lucia.morper-busch@uni-wuerzburg.de \\ ${ }^{2}$ Department of Geoinformatics - Z_GIS, University of Salzburg, Austria
}

Short paper

\begin{abstract}
The European (EC FP7-funded) HEALTHY FUTURES project aims at improving anticipation of climate change effects on three water related vector-borne diseases (malaria, schistosomiasis, and Rift Valley fever) in eastern Africa. The intention of the HEALTHY FUTURES Atlas is to present key project results, and to support decision makers in identifying context and place-specific interventions. The Atlas provides information on past, present, and future conditions of risk and its components (hazard, vulnerability) to the three diseases, and allows the exploration of results through interactive tools.
\end{abstract}

\section{Methods}

\subsection{Concept}

The HEALTHY FUTURES Atlas ${ }^{1}$ was designed to present the project results in a comprehensible and spatially explicit way to relevant target groups. It contains information on past disease outbreaks, as well as on current and future risk of infection in eastern Africa (TOMPKINS \& ERMERT 2013, KIENBERGER \& HAGENLOCHER 2014, MCCREESH et al. 2015). The results are portrayed as maps, with appropriate legends, while explorative tools enable an analysis of the underlying datasets. Historical data of the three target diseases was collected through extensive archival research in Africa and Europe; each entry of the historical database can be displayed with spatial and temporal filter options. Homogeneous regions of risk and social vulnerability are visualized and can be decomposed into their underlying factors. Future estimations of disease hazard (i.e. prevalence) integrate multiple ensembles of downscaled and bias-corrected climate projections based on two Representative Concentration Pathways (RCP4.5, RCP8.5). The decades from 2010 to 2090 can be sequentially explored, and temporal trends for single locations can be queried.

\subsection{Technical Implementation}

The HEALTHY FUTURES Atlas is designed as an open-source WebGIS using OGC (Open Geospatial Consortium) web mapping standards. The WebGIS architecture com-

\footnotetext{
${ }^{1}$ Accessible at www.healthyfutures.eu

GI_Forum - Journal for Geographic Information Science, 1-2015.

(c) Herbert Wichmann Verlag, VDE VERLAG GMBH, Berlin/Offenbach. ISBN 978-3-87907-558-4.

(c) ÖAW Verlag, Wien. ISSN 2308-1708, doi:10.1553/giscience2015s460.
} 
poses client, server, and data components (Fig. 1). The user's web browser acts as the client, while the server hosts the client application, the map server, and the data. The map server, which links the geodata and the client, is an open-source software (GeoServer), and provides map layers in the OGC data exchange format Web Map Service (WMS) and Web Feature Service (WFS). The vector geodata is stored in an open-source spatial database (PostGreSQL with spatial extension PostGIS), while the raster geodata lies in a server directory as GeoTIFF-files. The open-source OpenStreetMap is used as a background map.

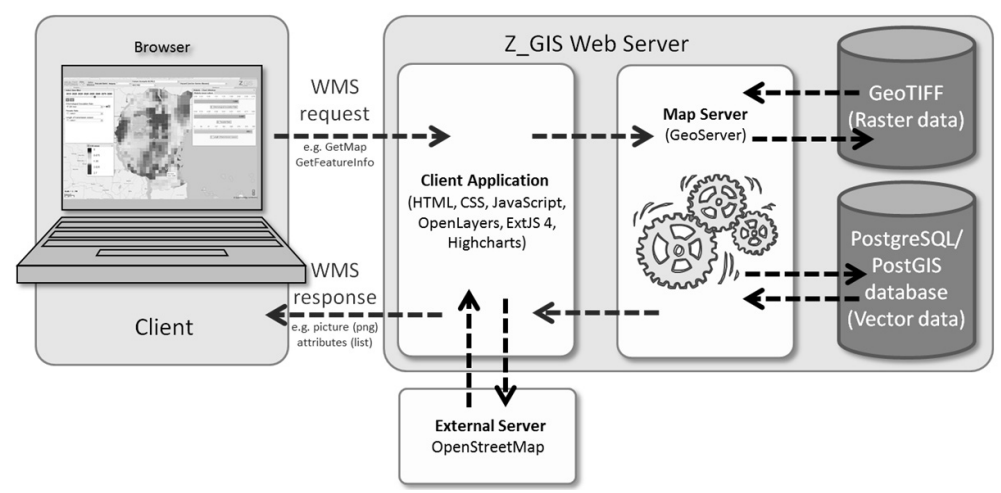

Fig. 1:

The WebGIS architecture with Client-ServerData structure

The client application is written in HTML, CSS, and the open-source JavaScript frameworks OpenLayers, ExtJS, and HighCharts. OpenLayers' mapping functionality is enhanced with Graphical User Interface (GUI) components of ExtJS and HighCharts, which are required for a demanding layout such as draggable windows, drop-down menus, time sliders, and charts. For the implementation of the complex concept outlined above, and to meet the needs of an intuitive usability, it is necessary to develop special features for which ready-to-use solutions cannot be found in predefined frameworks (MORPER-BUSCH et al. 2013).

\subsection{User Validation}

From the beginning of the design phase, a continuous exchange with possible users took place to ensure the best usability of the tool. Ideas and suggestions were implemented and then immediately evaluated and improved in an intensive iterative process.

A beta version of the HEALTHY FUTURES Atlas was presented to 20 potential users, accompanied by individual tests and an online survey. The tests were carried out using the "Think-aloud protocol" method during a stakeholder workshop in Nairobi, Kenya, where a person speaks aloud every action or insight while solving pre-defined tasks. Screen and voices were recorded during the test. This method makes it possible to follow the user's interaction with the Atlas, and to understand possible difficulties. The second part of the test, the online survey, allows the user's behaviour to be related to his/her knowledge and background. Moreover, with this survey, the users evaluated functions, design, and usability of the Atlas, directly after completing the given tasks. 


\section{Results}

To ease the use of the HEALTY FUTURES Atlas, an introductory window was created as a splash screen. It enables the user to select the desired options (disease, time scale, and risk component) by link-sensitive graphics (images with clickable areas). When all three options are chosen, the intro window disappears and the main map window appears.

The main map window consists of a full-screen map, a top toolbar, a selection window and a chart window that appears when a particular location is selected in the map. As in the intro window, three selection options are available in the toolbar. To save space, the clickable graphics are combined with drop down menus. They appear when the drop down is opened, and disappear when the selection is done.

The content of the selection window varies depending on the selected options. The main element is the map layer selection, where the user can select the desired layer either using checkboxes or a drop down menu. For the chosen map layer, the respective legend graphic is shown. When visualizing the historical data, additional filter options (based on attributes and time) for the clustered map points are integrated in the selection window.

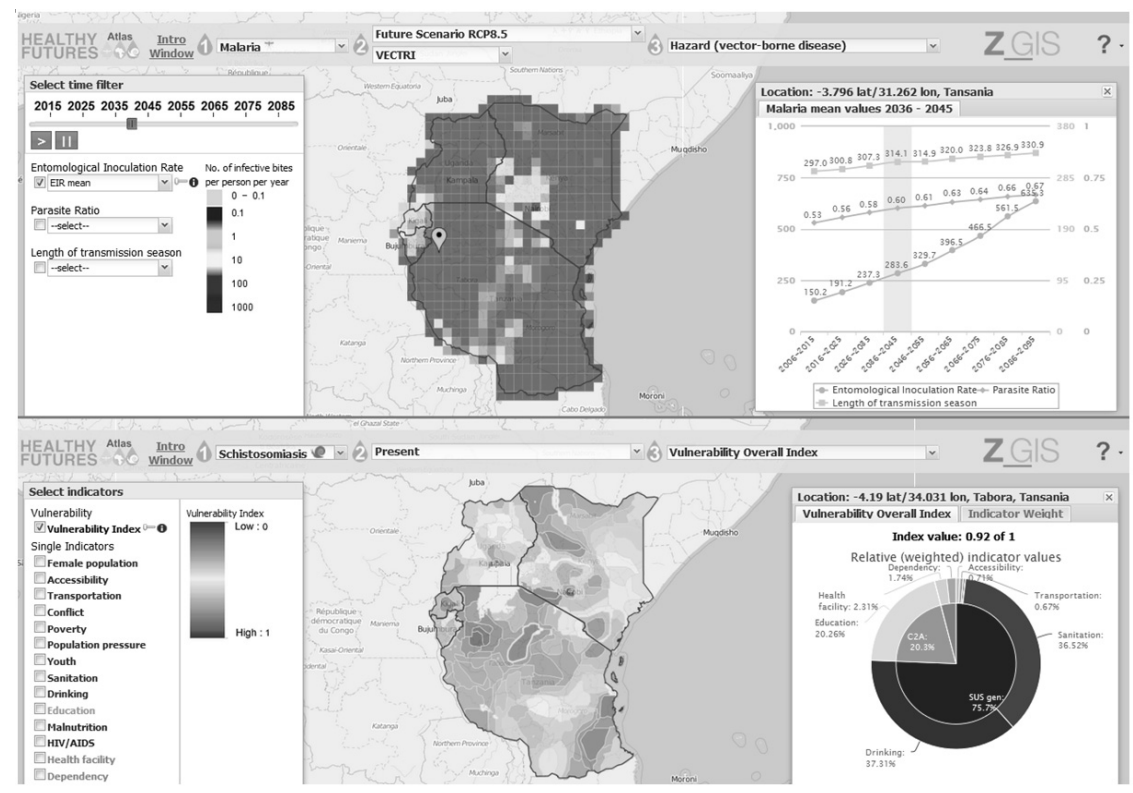

Fig. 2: Main map window of the HEALTHY FUTURES Atlas (here: future potential of infective malaria bites (above) and social vulnerability for schistosomiasis (below))

The time slider in the future option is connected with time-enabled WMS map layers, which are available for different decades ranging from 2010 to 2090 (Fig. 2 above). The corresponding chart displays values of all decades for one location with the selected decade highlighted. When the time slider is moved, both the map and the highlighted decade of the chart are adjusted. Alternatively, it is possible to turn on the time animation, which passes 
through the decade maps in a 1.5 second interval. By clicking on a map location, the chart window opens and shows pie, bar, or line charts, depending on the chosen option. Not only the value represented in the map (e.g. index) is displayed, but also e.g. underlying indicator values (vulnerability) (Fig. 2 below), or all available time periods (scenarios) (Fig. 2 above).

Summarizing the results of the user validation, the HEALTHY FUTURES Atlas satisfies most of the expectations of the test persons. Furthermore, they were surprised by the quantity of information and the breakdown of the underlying factors into a variety of maps and charts. Several enhancements have been suggested, some of which were implemented directly after the survey. Additional comparison and export functions as well as more background information were proposed to be included in further versions of the Atlas.

\section{Conclusion and Outlook}

The HEALTHY FUTURES Atlas is an important part of meeting the aims of the research project, since it has been designed to provide a meaningful overview of the several project results. The Atlas generated an added value for the project as the consolidation demand initiated a process of reconsidering and - where necessary - compiling the results which are relevant for permitting identification of hotspots for infectious disease risks under different climate change scenarios and at policy-relevant time-steps over this century throughout eastern Africa. In addition, the Atlas can be used to generate visualisation aids for incorporation into, e.g., policy and planning documents, and as part of a larger resource base to inform surveillance and intervention strategies. The HEALTHY FUTURES Atlas has already provided important input to the development of national multi-hazard early warning systems, and it is envisaged that the tool will be further developed.

\section{References}

Kienberger, S. \& Hagenlocher, M. (2014), Spatial-explicit modeling of social vulnerability to malaria in East Africa. International Journal of Health Geographics, 13 (29).

McCreesh, N., Nikulin, G. \& Booth, M. (2015), Predicting the effects of climate change on Schistosoma mansoni transmission in eastern Africa. Parasit Vectors, 8 (1).

MorPer-Busch, L., Bertermann, D., Klug, H., Bialas, C., Orosz, L. \& SimÓ, B. (2013), ThermoMap - An Open Source Web Mapping Solution for Visualising Very Shallow Geothermal Energy Potentials. ICC 2013 Conference Proceedings.

TOMPKINS, A. M. \& ERMERT, V. (2013), A regional-scale, high resolution dynamical malaria model that accounts for population density, climate and surface hydrology. Malaria Journal, DOI: 10.1186/1475-2875-12-65.

\section{Acknowledgments}

The research leading to these results has received funding from the European Union's Seventh Framework Programme (FP7/2007-2013) under grant agreement no. 266327 (HEALTHY FUTURES, http://www.healthyfutures.eu/). 